ISSN 1112-9867

Available online at http://www.jfas.info

\title{
LABORATORY CLINICAL ASSESSMENT OF PATIENTS WITH AML OVER SIX YEARS (2010-15) IN HEMATOLOGY - ONCOLOGY OF NAMAZI HOSPITAL IN SHIRAZ
}

\author{
H. A. Rostami, Z. Rahmanian
}

Department of internal medicine, Jahrom University of medical sciences, Jahrom, Iran

Published online: 15 May 2016

\begin{abstract}
Introduction: Acute myeloid leukemia is a group of neoplasm of the bone marrow that hasclinical similarity but has but a specific cellular morphology and genetics.AML includes about $90-80 \%$ of adult acute leukemia and is the third leading cause of death due to cancer in people aged 30-18 years old. So, this study aims to examine patients with AML in a clinical laboratory manner who have been diagnosed over six years (2010-15) in Hematology-Oncology section of Namazi hospital and admitted there.
\end{abstract}

Method: By using acute myeloid leukemia patients' records who had been hospitalized six years (94-89) in Hematology-Oncology section of Namazi hospital, this study was performed retrospectively.The necessary data included age, sex, diagnosing leukemia type, place of birth, occupation, time of admission and discharge, duration of hospitalization, the patient's chief complaint and other complaints, the duration of the preliminary symptoms prior to diagnosis, history of smoking and exposure to radiation or chemicals, physical examination findings and laboratory findings including blood chemistry and bone marrow, study of cerebrospinal fluid in case it had been done, positive culture and information relating to the treatment of early response, duration of remission, complications of induction of remission and the time of

Author Correspondence, e-mail: author@yahoo.com_

doi: http://dx.doi.org/10.4314/jfas.v8i3s.282 
recurrence and death and the cause of death, all of which were recorded in a form that was attached. The results of this study are indicated in an observational way. But, wherever it was possible, comparison was done and this comparison was evaluated using $\mathrm{Z}$ test. $\mathrm{P}<0.05$ is significant.

Results: During this period, 244 patients were diagnosed and hospitalized that 141 of them (58\%) were male and 103 (42\%) were female. Then, M1 with (20\%), M2 (6/19\%) and M3 with $18.3 \%$ are ordered. Finally M5 with 8\%,and M7 and Hybrid each with $1.2 \%$ and M6 with $0.8 \%$ are classified in the next ranks.Most patients were from the age group of 29-20 years. Regardless of the type of leukemia and sex,the average age of our patients was 36/17 years (SD 16/11 \pm years). The worst prognosis of M3 was death initial response to chemotherapyin $52.5 \%$ and the best prognosis of M2 was death as the initial response to treatment in $27.5 \%$. The most common cause of death in our patients was first bleeding with $38.5 \%$ and then infection with $27 \%$.

Conclusion: Acute myeloid leukemia, unlike in other parts of the world,is still a lethal disease with anominous sequel among our young population. Swollen lymph glands and organs are more common in patients. A closer examination and laboratory examination, at least a complete blood test in suspected patients could be life-saving.

Keywords: AML, Hematology, Oncology.

\section{INTRODUCTION}

The presence of genetic factors in A.N.L.L based on the observation of an increased risk of A.N.L.L in patients is associated with congenital defectssuch as Down syndrome and Klinefelter. The risk of leukemia in Down syndrome is ten times higher than the normal population [12].Diseases associated with chromosomal instability and increased chromosome breakage include Fanconi anemia, ataxia telangiectasia, Bloom syndrome and Vyskvt Aldrich [4-5], that are coupled with the increased prevalence of A.N.L.L; and diseases like One Rklyng Hazvn and congenital neurofibromatosis are associated with an increased prevalence of C.L.L in childhood [3]. Environmental factors are also involved in the pathogenesis of AML [6]. There are only three environmental factors that are well known as causes of AML including:getting too much radiation, chronic exposure to benzene and Lkalyt [3]. Evidence on the association of leukemia and radiation in Hiroshima and Nagasaki atomic bomb survivors has been seen. The time its incidence was from 5 to 21 years after the explosion. Risk depends on age at the time of the 
explosion and the dose of radiation [2, 4]. The highest incidence was among those under 10year-old and 50-year-old [2]. Symptoms of acute leukemia in most patients are a period of less than three months [5]. Signs and symptoms that show the onset of AML include pallor, weakness, fatigue, palpitations and dyspnea on exertion which indicates anemia. However, weakness and feeling of well-being cannot be linked to the severity of anemia. Bruising due to trivial shot, palpitation, epistaxis, gingival bleeding and prolonged bleeding from skin lesions, indicative of thrombocytopenia, are among the initial manifestations of the disease. Incidence of bleeding from the stomach, intestines, genital tract, lungs or central nervous system, though low, can be a part of the initial manifestations [3].

\section{METHOD}

By using acute myeloid leukemia patients' records who had been hospitalized six years (94-89) in Hematology-Oncology section of Namazi hospital, this study was performed retrospectively. According to the list Archive of Shahid Motahhari clinic, 352 people were hospitalized due to AML during this period,244 of whom were aged 14 years or older and had been diagnosed for the first time.The necessary data included age, sex, diagnosing leukemia type, place of birth, occupation, time of admission and discharge, duration of hospitalization, the patient's chief complaint and other complaints, the duration of the preliminary symptoms prior to diagnosis, history of smoking and exposure to radiation or chemicals, physical examination findings and laboratory findings including blood chemistry and bone marrow, study of cerebrospinal fluid in case it had been done, positive culture and information relating to the treatment of early response, duration of remission, complications of induction of remission and the time of recurrence and death and the cause of death, all of which were recorded in a form that was attached. Unfortunately, because of incomplete records available including not having writtenpatients' job or restricting the report to mentioning jobs in general, say writing only "worker", regardless of the place of work, the relationship between occupation and type of leukemia rendered impossible.Moreover, the relationship between smoking and HDL in prognosis and the risk of leukemia was not statistically significant,so they were excluded from the study. The rest of the data were analyzed after extraction. The results of this study are indicated in an observational way. But, wherever it was possible, comparison was done and this comparison was evaluated using $\mathrm{Z}$ test. $\mathrm{P}<0.05$ is significant.Concerning the median duration of 
remission and survival and the duration of initial symptoms of leukemiaprior tothe diagnosis because data range was too broadand the standard deviation was larger than the average in these three cases,some maximum and minimum data were deleted to modify the variance.

$Z=\frac{\overline{p-p_{0}}}{\sqrt{\frac{p_{0}\left(1-p_{0}\right)}{n}}}$

\section{RESULTS}

Of 244 AML patients that were studied, 141 (58\%) were male and $103(42 \%)$ were female. Of 244 patients that were studied, 49 people had acute myeloid leukemia M1 (20\%), 48 had type M2 (19.6\%), 46 had M3 (18.8\%), 73 had M4 (29.9\%), 20 had M5 (8\%), 2 had M6 (0.8\%), 3 had two-phenotype type (1.2 percent).

Table 1: Mean age of AML patients based on gender and type of leukemia:

Table 1:

\begin{tabular}{|l|l|l|l|l|l|l|}
\hline $\begin{array}{l}\text { Average } \\
\text { age(Year) }\end{array}$ & Total & $\begin{array}{l}\text { Average } \\
\text { age(Year) }\end{array}$ & Female & $\begin{array}{l}\text { Average } \\
\text { age(Year) }\end{array}$ & Male & $\begin{array}{l}\text { Gender } \\
\text { Type }\end{array}$ \\
\hline 36.875 & 49 & 33.7 & 18 & $38 / 76$ & 31 & M1 \\
\hline 36.57 & 48 & 35.9 & 21 & 37.7 & 27 & M2 \\
\hline 29.55 & 46 & 29.55 & 20 & 30 & 26 & M3 \\
\hline 39.27 & 73 & 36.76 & 30 & 41.07 & 43 & M4 \\
\hline 41 & 20 & 38.8 & 10 & 43.2 & 10 & M5 \\
\hline 44 & 2 & 28 & 1 & 60 & 1 & M6 \\
\hline 38.66 & 3 & 19 & 1 & 48.5 & 2 & M7 \\
\hline 31.66 & 3 & 39 & 2 & 39 & 1 & Hybrid \\
\hline
\end{tabular}


Table 2: Frequency of AML patients in 1990-95 in terms of sex and place of residence:

\begin{tabular}{|l|l|l|l|l|l|l|}
\hline Percent & Total & Percent & Female & Percent & Male & $\begin{array}{l}\text { Gender, } \\
\text { Percent } \\
\text { province }\end{array}$ \\
\hline 68 & 165 & 41 & 67 & 59 & 98 & fars \\
\hline 10 & 24 & 58 & 14 & 42 & 10 & Kerman \\
\hline 7 & 18 & 33 & 6 & 67 & 12 & Bushehr \\
\hline 6.9 & 17 & 35 & 6 & 65 & 11 & $\begin{array}{l}\text { Kohgiluyeh } \\
\text { and Boyer- } \\
\text { Ahmad }\end{array}$ \\
\hline 3.6 & 9 & 33 & 3 & 67 & 6 & Khuzestan \\
\hline 2.9 & 7 & 71 & 5 & 29 & 2 & Hormozgan \\
\hline 1.6 & 4 & 50 & 2 & 50 & 2 & The Rest \\
\hline 100 & 244 & 42 & 103 & 58 & 141 & Total \\
\hline
\end{tabular}

165 AML patients were from Fars province that Shiraz, with 60 patients (36\%), had the highest number of the patients referring to hematology-Oncologysection of Namazi hospital. And Shiraz, cities such as Marvdasht with 16 patients (9.7\%), Jahrom with 14 patients (8.5\%), Karzerun with 9 patients (5.4\%), Fasawith 8 patients $(4.8 \%)$ and etc. were listed. 
Table 3: The main complaint of AML patients during 94-89 years:

\begin{tabular}{|l|l|l|}
\hline Percent & Number & $\begin{array}{l}\text { The number of major } \\
\text { complaint }\end{array}$ \\
\hline 31 & 76 & Fever \\
\hline 18 & 43 & General Weakness \\
\hline 17 & 42 & Bleeding Signs \\
\hline 16 & 39 & Fatigue \\
\hline 11 & 27 & Wanness \\
\hline 7 & 17 & Gingival Bleeding \\
\hline 5 & 12 & General Pain \\
\hline 4.5 & 11 & Headache \\
\hline 1.6 & 4 & Anorexia \\
\hline 1.2 & 3 & Beech \\
\hline 0.8 & 2 & Weight Loss \\
\hline
\end{tabular}

\section{DISCUSSION}

In this study, $141(58 \%)$ of the patients were male and $103(42 \%)$ are female.Unlike survey conducted by doctor Mahallati, the ratio of men to women was much higher than the reported statistics. Now, the male to female ratio is $1 / 36$. Like other studies, it is slightly more in men than in women. With little disturbance,this increase can be achieved in a variety of acute myeloid leukemia.The prevalence of different types of acute myeloid leukemia is M2 (30-25\%), M4 (3020\%), M1 (20\%), M3 (10-5\%), M5 (10\%), M6 and M7, respectively, each 5\% [2].According to the conducted studies, the most common type was M4 with 73 patients that accounts for $29.9 \%$ of all cases which is statistically meaningfulby $\mathrm{P}<0.0001$.After M4, type M1 with 49 patients (20\%) about $\mathrm{P}<0.05$, M2 with 48 patients (19.6\%) stand out.The high prevalence of M3 with 46 cases $(18.8 \%)$ has a significant increase in comparison with other studieswhich is statistically significant $\mathrm{P}<0.0001$. The percent of our M6 and M7 patients was much lower compared with other statistics. The prevalence of both types of leukemia is 5\%, and according to our study, M6 
is 0.8 and $\mathrm{M} 7$ is $(1.2 \%)$ and this is not statistically significant because the number of patients in the two groups is too low. According to our study, the percent of M5 is 8\% which does not show any significant difference with the statistics of other studies. The studied patients aged 14-80 years old.The average age of men with AML ismore than the average age of women who are suffering AML (37/35 years against 34/3 years)and this ratio is maintained in all types of AML. It is a matter of controversy that why women in younger age,compared to men, are diagnosed with AML. M3 patients are younger than other types of AML [7] and this is the case with our patients. The age of M6 patients is usually higher and their average age is more than 50 years [23]. Likewise in the conducted studies, the mean age of M6patients is higher than with other types of AML. But because the number of patients was small, it is statistically not significant.Age distribution of the studied patients was categorized from 14 to 70 and then compared. The highest percent (22/13) was ascribed to the age group of 29-20 years and then to 39-30 years age group (21/31\%).Unlike other studies in whichits prevalence increases with an increase in age [2, 3, 5 and 8] and half of AML patients areover 60 years of age [5], in our patients, its prevalence decreases with an increase in age. This is probably due to lower life expectancy and lower average age of our population in comparison with the studied populations.Detection of new cases of AML increases from1990 to 1995.So that $9 \%$ of patients were diagnosed in1990and 21\%of them were diagnosed in 1994. This increase was maintained in 1995, and this may indicate acute myeloid leukemia incidence in our population due to industrialization, air pollution or changes in eating habits and in the people's lifestyle. Such as increaseduring 1965-75 in the UK had been reported in studiesand AML incidence had doubled over these years [6].Infection is the complicationof almost all acute leukemia [5].In most patients there is a fever which is due to either leukemia or secondary infection $[2,9]$.The major complaint of our patients was to have a fever.It accounted for $32 \%$ of the major complaint and the reason of theirreferring and in examining the history, $67 \%$ of patients complained of fever or had a febrile history. Weight loss, which is usually not severe, is seen in 50\% of patients [7]. But in this study, only $36 \%$ of patients had a history of weight loss. And the major complaint of only $0.8 \%$ of our patients was weight loss.And this is statistically significant $(\mathrm{P}<0.0001)$. Bleeding is a major issue in patients with acute leukemiaand is mainly related to thrombocytopenia [5]. Thrombocytopenia is almost always in diagnosis which is due to reduced production and low life-expectancy.Half of the patients at the time of platelets diagnosis have less than 50 thousand per microliter [3].121 of our 
patients $(50 \%)$ had signs of bleedingwhile 159 cases $(65 \%)$ had a platelet count less than 50 thousand per microliter. And this is much higher than other conducted studies $\mathrm{P}<0.0001$ which is statistically significant.In this study, the relationship between age and the risk of leukemia, sex and type of leukemia, location and type of leukemia was examined none of which was statistically significant. Furthermore, the relationship between age and the type of leukemia, residence location and prognosis was studied that was not statistically significant.

\section{CONCLUSION}

In our population like the rest of the world, AML disease is more common in men than women, and this proportion is maintained in all types of AML.Here, the prevalence of acute myeloid leukemia in order of frequency is M2, M1, M4 and then M5, M3and finally HybridM7 and M6 come which is unlike the statistics of other studies in which M2 is the most common type and this may be due to the similarity of the two morphologies. The average age of patients with AML in our population is much lower than similar studies in other countries.AML age distribution of our population is also against the statistics from other studies.It decreases with an increase in the age of the patients. And most patients in our study are 29-20 year-olds and then those who are 30 to 39 years old. And this is the antithesis of the available statistics that state this disease is less in early adulthoodand it is more common with increasing age. A small percentage of our patients are elderly patientswhich may indicate that our population is young and lower life expectancy in our population.

\section{REFERENCES}

1. Taub. JW, Yaddarapudi, Ravindranath: Treatment of childhood AML. Baillier'sclin-hematol. 9:129-146, 1996.

2. Miller K.B: clinical manifestation of AML in: Haffman'shematology basic principles and practice 2nd ed. Churchill livingstoninc. 1995

3. Lichtman M.A: AML in: william's Textbook of hematology $5^{\text {th }}$ edition litchman.co. 1995

4. Leeuwen F.V: Risk of AML and MDS following cancer treatmentin: Bailliere'sclin. hematol. 9:57-85, 1996

5. Scheinbrg D.A, Goide D.W: the leukemia in Harrison's principles of internal medicin $13^{\text {th }}$ ed. MC Grawhillinc 1994. 
6. Rohantiner A, lister T.A: Acute myelogenous leukemia in adult in:leukemia 6th ed. W.B saunders 1996

7. Fenaux P., Degos L: Treatment of acute promyelocytic leukemia in Bailliere'sclin. Hematol 6:107-128:1996.

8. Rowe JM, Liesweld JL: Treatment and prognostic factor in AMLin: Bailliere'sclin-hematol. 9:87-105, 1996

9. Greer J.P. and Kinney M.C: ANLL in wintrobe clinical hematology $9^{\text {th }}$ ed. Lea \&Febiger. 1993.

How to cite this article:

Rostami H.A, Rahmanian Z. Laboratory clinical assessment of patients with aml over six years (2010-15) in hematology - oncology of namazi hospital in shiraz. J. Fundam. Appl. Sci., 2016, $8(3 S), 1012-1020$. 